\title{
QTLs for days to silking in a recombinant inbred line maize population subjected to high and low nitrogen regimes
}

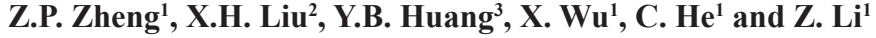 \\ ${ }^{1}$ Nanchong Institute of Agricultural Sciences, Nanchong City, P.R. China \\ ${ }^{2}$ Key Laboratory of Southwest China Wildlife Resources Conservation, \\ Ministry of Education, College of Life Sciences, \\ China West Normal University, Nanchong City, P.R. China \\ ${ }^{3}$ Maize Research Institute, Sichuan Agricultural University, \\ Ya'an City, P.R. China \\ Corresponding author: X.H. Liu \\ E-mail: liuxiaohong0817@yahoo.com.cn
}

Genet. Mol. Res. 11 (2): 790-798 (2012)

Received January 1, 2011

Accepted October 10, 2011

Published April 3, 2012

DOI http://dx.doi.org/10.4238/2012.April.3.1

\begin{abstract}
Days to silking (DTS) is one of the most important traits in maize (Zea mays). To investigate its genetic basis, a recombinant inbred line population was subjected to high and low nitrogen $(\mathrm{N})$ regimes to detect quantitative trait loci (QTLs) associated with DTS. Three QTLs were identified under the high $\mathrm{N}$ regime; these explained $25.4 \%$ of the phenotypic variance. Due to additive effects, the QTL on chromosome 6 increased DTS up to 0.66 days; while the other two QTLs mapped on chromosome 9 (one linked with Phi061 and the other linked with Nc134) decreased DTS 0.89 and 0.91 days, respectively. Under low $\mathrm{N}$ regime, two QTLs were mapped on chromosomes 6 and 9, which accounted for $25.9 \%$ of the phenotypic variance. Owing to additive effects, the QTL on chromosome 6 increased DTS 0.67 days, while the other QTL on chromosome 9 decreased it 1.48 days. The QTL on chromosome 6, flanked by microsatellite markers Bnlg1600 and Phi077, was detected
\end{abstract}


under both $\mathrm{N}$ regimes. In conclusion, we identified four QTLs, one on chromosome 6 and three on chromosome 9. These results contribute to our understanding of the genetic basis of DTS and will be useful for developing marker-assisted selection in maize breeding programs.

Key words: Maize (Zea mays L.); Days to silking; Nitrogen regime; Quantitative trait locus; Recombinant inbred line

\section{INTRODUCTION}

Nitrogen $(\mathrm{N})$ is a very important element in plants, where it is related to growth and development. However, at present, most maize (Zea mays L.) in developing countries is produced under $\mathrm{N}$-deficient conditions because of low $\mathrm{N}$ use efficiency, limited availability of fertilizer, or low purchasing power of farmers (Bänziger et al., 1997; Agrama et al., 1999). N deficiency can severely affect many metabolic pathways and physiological progresses in maize (Ribaut et al., 2007), and a change in the number of days to silking (DTS) is one of the most serious consequences.

A too-short DTS will shorten maize growing time and probably bring about yield loss. On the other hand, a too-long DTS will prolong growing time and this also leads to some problems, where low-temperature stress in the maize growing stage is a typical instance in Northern China (Yang et al., 2007). One of the effective ways to resolve this problem is to breed the varieties with ideal DTS, but the conventional breeding method is very time-consuming and laborious. An alternative solution is to utilize the elite genes controlling DTS to improve the character of long- or short-DTS cultivars, and the basic work is to determine the genetic basis of DTS. QTL mapping is an efficient approach to elucidate genetic basis (Sabadin et al., 2008).

Some QTLs affecting DTS have been mapped (Ribaut et al., 1996; Tang et al., 2005; Szalma et al., 2007). However, different experimental environments have produced different results, such as QTL number, position or genetic effects. For example, using $180 \mathrm{~F}_{2: 3}$ families derived from an L050 $\times$ B73 cross, Zhang et al. (2004) detected four QTLs on chromosomes $1,2,3$, and 7 under a well-watered environment, while under drought-stress environment, only two QTLs were mapped on chromosomes 3 and 6. According to the published literature, previous studies of ecological conditions designed for QTL mapping for DTS focused on the differences in water content in soil (Ribaut et al., 1996; Zhang et al., 2004; Wu et al., 2008), whereas different $\mathrm{N}$ regimes have hardly been used to date (Ribaut et al., 2007).

Although previous studies of populations for QTL mapping associated with DTS focused on $\mathrm{F}_{2}$ (Khairallah et al., 1998; Zhang et al., 2004; Tang et al., 2005; Gong et al., 2006; Ribaut et al., 2007; Hu et al., 2008), this kind of segregating population was temporary, because no continued plants were available for DNA extraction and phenotypic analysis. However, the recombinant inbred line (RIL) population is immortal, due to homozygous individuals, and could be used in different regions and times (Liu et al., 2010). At present, the RIL population has been widely used to identify QTLs in plant (Wan et al., 2006; McIntyre et al., 2010; Yang et al., 2010). However, up to now, only a few studies on QTL mapping for maize DTS using the RIL population have been reported.

Therefore, in the present study, the QTLs for DTS were mapped and characterized using an RIL population under different $\mathrm{N}$ regimes. The main objectives were to 1 ) identify and compare the QTLs controlling DTS, and 2) look for the markers to be used for markerassisted selection (MAS). 


\section{MATERIAL AND METHODS}

\section{Plant materials}

The experimental materials involved in this study included maize inbred lines Mo17 (long DTS) and Huangzao4 (short DTS), $\mathrm{F}_{1}$ and an $\mathrm{F}_{9}$ RIL population consisting of 239 RILs. Mo17 and Huangzao4 are the representative lines of Lancaster and Tangsipingtou heterotic groups, respectively, and $\mathrm{F}_{1}$ and the RIL population were derived from the cross between the two parental lines Mo17 and Huangzao4.

\section{Experimental measurement and phenotypic observation}

Parental lines, $F_{1}$ and the RIL population were sown in a randomized complete block design with six replicates at the experimental farm of Nanchong Institute of Agricultural Sciences, Nanchong City, P.R. China. Single-plant planting was used with 15 plants per replicate, of which three replicates were under high $\mathrm{N}$ regime (HNR) by applying urea, $300 \mathrm{~kg} /$ ha, and another three replicates were under low $\mathrm{N}$ regime (LNR) with no added $\mathrm{N}$ fertilizer. The average contents of total $\mathrm{N}$ and alkaline hydrolysis $\mathrm{N}$ in $30-\mathrm{cm}$ depth soil were 0.092 and $0.000056 \%$, respectively.

At the time of flowering, eight plants in the middle of every replicate were individually investigated for the trait DTS, based on the data of the RIL population. The SPSS11.5 software (www.spss.com) was used for descriptive statistics, analysis of variance (ANOVA) and correlation analysis of the trait DTS.

\section{QTL mapping}

Based on the data for DTS in the RIL population and the established genetic map consisting of 100 SSR markers and covering $1421.5 \mathrm{cM}$ of mapping distance (Liu et al., 2009), the QTL(s) controlling DTS under two N regimes were analyzed separately by the composite interval mapping (CIM) method of the Windows QTL Cartographer 2.5 software (Wang et al., 2010), with a scanning interval of $2 \mathrm{cM}$ between markers and putative QTLs and a window size of $10 \mathrm{cM}$. The LOD ( $\log 10$ of odds ratio) threshold value for the QTL significance was determined by a 1000-time permutation test $(\alpha=0.05)$ (Doerge and Churchill, 1996). The cofactors used for calculation of CIM were selected by the program using forward stepwise regression, and LOD curves were created by scanning all linkage groups. The QTLs with an LOD value greater than the threshold value will be presented, and their position, genetic effects and percentage of phenotypic variation were estimated at the significant LOD peak in the region. The QTLs identified under the two $\mathrm{N}$ regimes were mapped with the Mapchart 2.1 software (Voorrips, 2002).

\section{RESULTS}

\section{Phenotypic observation and statistical analysis}

The results regarding DTS showed variations between the lines tested. With respect 
to the three lines Mo17, Huangzao4 and $\mathrm{F}_{1}$, Mo17 had the highest values under both $\mathrm{N}$ conditions, followed by Huangzao4 (Table 1). For the RIL population, the valid 237 RILs under high and low $\mathrm{N}$ regimes displayed differences at the 0.01 probability level (Table 2). Nevertheless, the data for the two groups showed a significant positive correlation at the 0.01 probability level, with a correlation coefficient of 0.895 .

Table 1. The phenotypic values of parental lines and $\mathrm{F}_{1}$ for days to silking.
\begin{tabular}{lccc}
\hline Nitrogen regime & M017 & HZ4 & $\mathrm{F}_{1}$ \\
\hline High nitrogen & 75.7 & 69.0 & 66.0 \\
Low nitrogen & 73.0 & 67.7 & 65.3 \\
\hline
\end{tabular}

$\mathrm{HZH}=$ Huangzao4.

Table 2. ANOVA of the recombinant inbred line (RIL) population on DTS under two nitrogen (N) regimes.

\begin{tabular}{llcrrrc}
\hline N regime & Variation source & Sum of squares & d.f. ${ }^{\text {a }}$ & Mean square & F & P \\
\hline High N & Between groups & 5665.55 & 236 & 24.01 & $8.44^{* *}$ & $<0.01$ \\
& Within groups & 1345.83 & 4723 & 2.85 & & \\
Low N & Between groups & 5694.15 & 236 & 24.13 & $14.34^{* *}$ & $<0.01$ \\
& Within groups & 794.00 & 472 & 1.68 & & \\
\hline
\end{tabular}

aThere were two missing values among the RIL population consisting of 239 RILs. ** Significant difference at 0.01 probability level. d.f. $=$ degrees of freedom.

The results of the descriptive statistics for the RIL population are shown in Table 3, and among the eight statistical parameters, all of them except standard deviation (SD) and skewness showed higher values for the RIL population under HNR than under LNR. In addition, from the frequency distribution graphs of the RIL population under two $\mathrm{N}$ regimes (Figures 1 and 2), it was found that the data of the two groups could agree with normal distribution, which suggests that the trait DTS was quantitative and controlled by multiple genes.

\begin{tabular}{|c|c|c|c|c|c|c|c|}
\hline $\mathrm{N}$ regime & Range & Minimum & Maximum & Mean & SD & Skewness & Kurtosis \\
\hline High N & 16.00 & 65.33 & 81.33 & 72.51 & 2.83 & 0.11 & -0.28 \\
\hline Low N & 13.33 & 64.67 & 78.00 & 71.15 & 2.84 & 0.24 & -0.44 \\
\hline
\end{tabular}

\section{QTL mapping}

The permutation test showed that the LOD threshold should be set at 3.16 and 2.89 for QTL identification under HNR and LNR, respectively. Under the conditions of the LOD threshold values, three QTLs were identified under HNR on chromosomes $6(Q 6 a-h n)$ and 9 ( $Q 9 a-h n$ and $Q 9 b-h n$ ) (Figure 3). They could account for 5.34, 9.67 and $10.36 \%$ of phenotypic variances, respectively (Table 4), and due to the additive effect, Q6a- $h n$ could increase DTS about 0.66 , while $Q 9 a-h n$ and $Q 9 b-h n$ could decrease DTS to some extent. Under LNR, only two QTLs were detected, one on chromosome $6($ Q $6 a$-ln $)$ and the other on chromosome 9 $(Q 9 c-l n)$. The two QTLs could explain 5.53 and $20.34 \%$ of phenotypic variance, respectively. The QTL on chromosome 6 had a positive additive effect (0.67), while the QTL on chromosome 9 showed a negative additive effect (-1.48). 


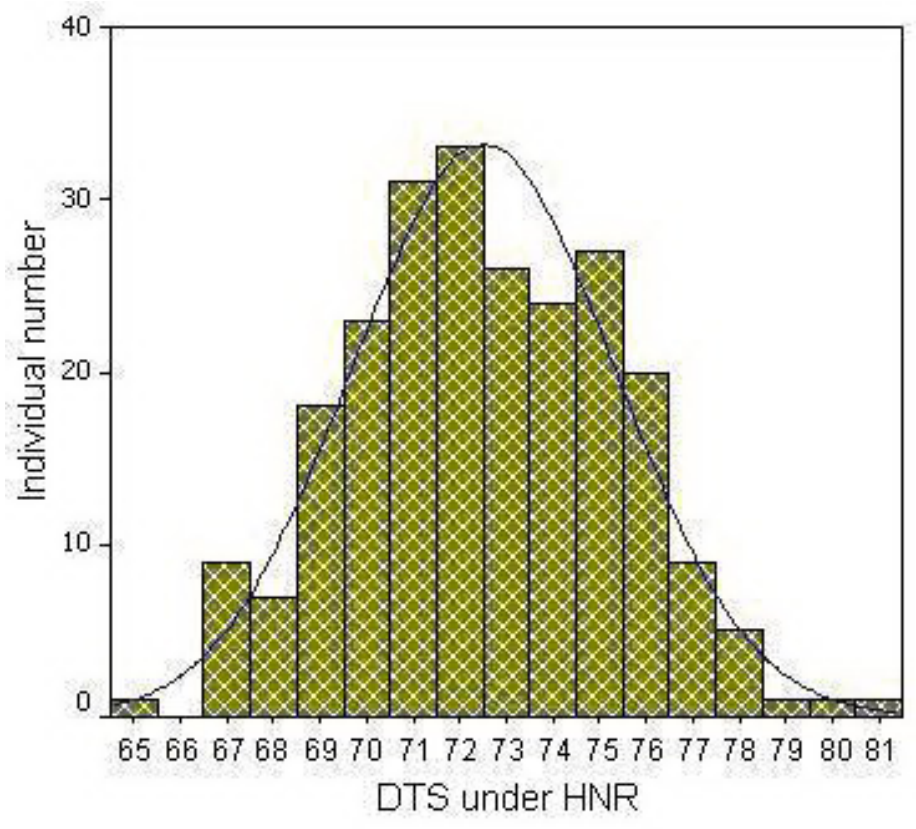

Figure 1. Frequency distribution graph of the recombinant inbred line population for days to silking (DTS) under high nitrogen regime (HNR).

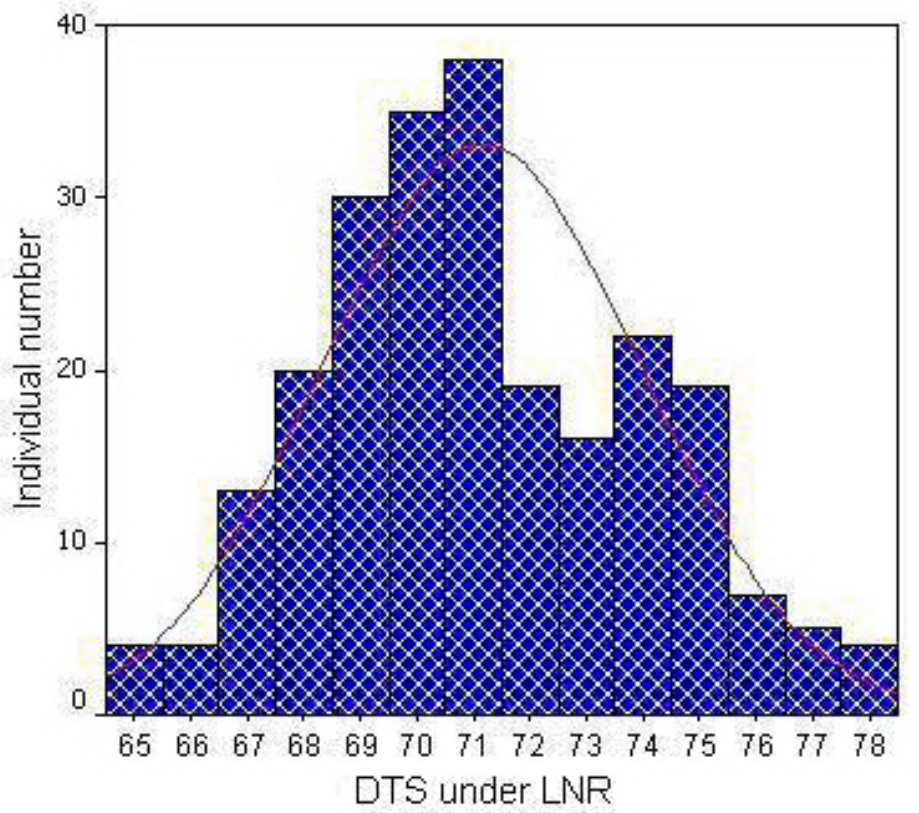

Figure 2. Frequency distribution graph of the recombinant inbred line population for days to silking (DTS) under low nitrogen regime (LNR). 


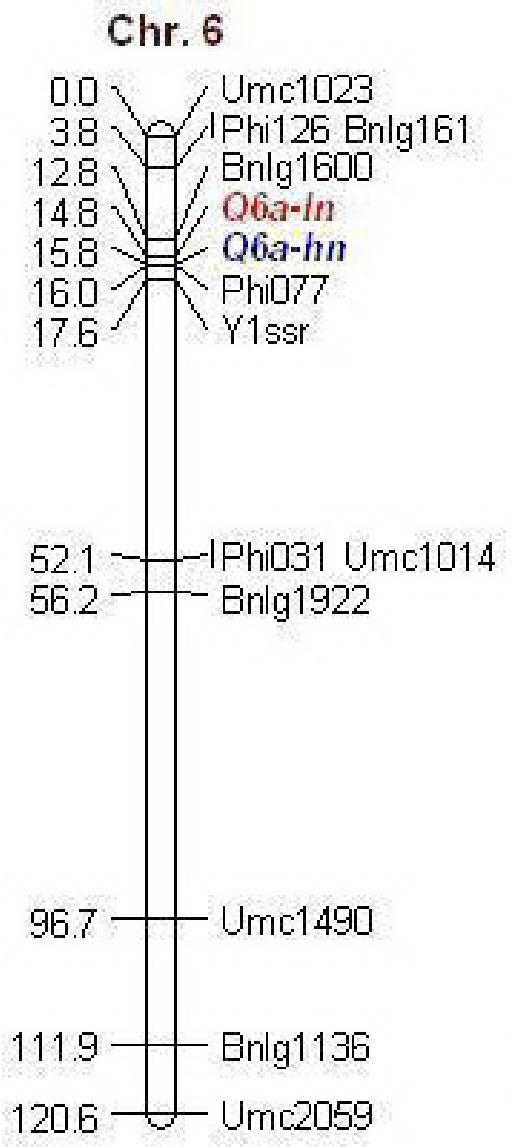

Chr. 9

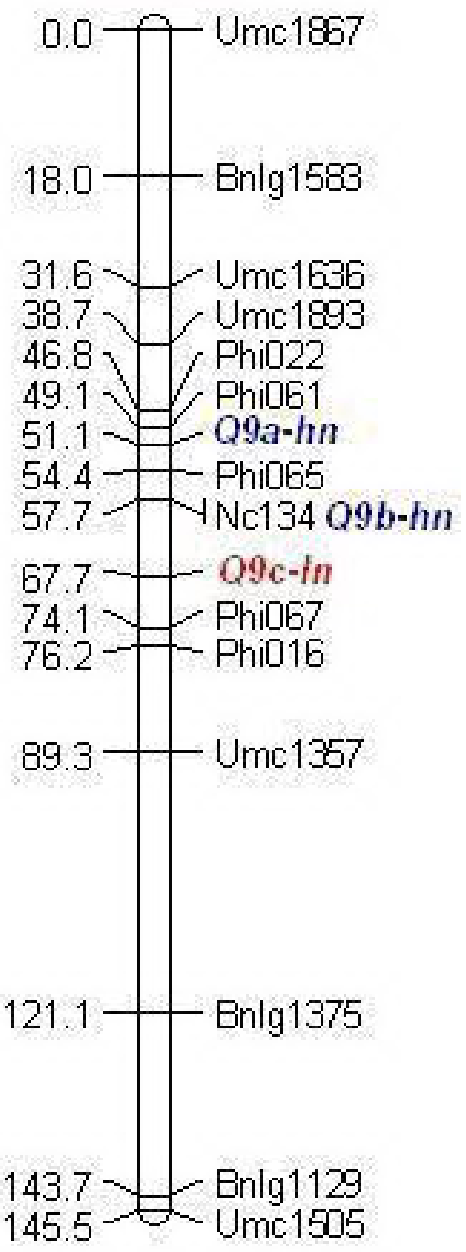

Figure 3. The chromosomal positions of the QTLs for DTS identified using the RIL population under two nitrogen (N) regimes. The three QTLs $Q 6 a-h n, Q 9 a-h n$ and $Q 9 b-h n$ were detected under high N regime (blue), while the two QTLs $Q 6 a-\ln$ and $Q 9 c-l n$ were detected under low N regime (red).

\begin{tabular}{|c|c|c|c|c|c|c|c|}
\hline $\mathrm{N}$ regime & QTL & Chr. & $\begin{array}{l}\text { Flanking markers } \\
\text { (Position, cM) }\end{array}$ & $\begin{array}{l}\text { Position } \\
(\mathrm{cM})\end{array}$ & LOD & $R^{2}(\%)$ & $\begin{array}{l}\text { Additive } \\
\text { effect }\end{array}$ \\
\hline \multirow[t]{3}{*}{ High N } & Q6a-hn & 6 & Bnlg1600-Phi077 & 15.8 & 3.19 & 5.34 & 0.66 \\
\hline & Q9a-hn & 9 & Phi061-Phi065 & 51.1 & 4.84 & 9.67 & -0.89 \\
\hline & Q9b-hn & 9 & $\mathrm{Nc} 134$ & 57.7 & 5.10 & 10.36 & -0.91 \\
\hline \multirow[t]{2}{*}{ Low N } & Q6a-ln & 6 & Bnlg1600-Phi077 & 14.8 & 3.12 & 5.53 & 0.67 \\
\hline & $Q 9 c-\ln$ & 9 & Nc134-Phi067 & 67.7 & 5.17 & 20.34 & -1.48 \\
\hline
\end{tabular}

$\mathrm{LOD}=\log 10$ of odds ratio. 
According to the chromosomal position and genetic effects of QTLs, it was concluded that Q6a-hn and Q6a-ln should be the same, detected under both N regimes. So in this study, there should be four QTLs that were detected under HNR and LNR, with one and three on chromosomes 6 and 9 , respectively.

\section{DISCUSSION}

$\mathrm{N}$ deficiency can affect many metabolic pathways and physiological progresses in maize (Ribaut et al., 2007), and a decrease in DTS is one of the most serious consequences. In this study, we investigated the genetic basis of DTS in maize using an RIL population and two N regimes to detect the QTLs controlling DTS. The results show that three and two QTLs were detected under HNR and LNR, respectively, of which the QTL on chromosome 6 was identified under both $\mathrm{N}$ regimes.

Compared to previous studies on QTL mapping for DTS (Khairallah et al., 1998; Zhang et al., 2004; Tang et al., 2005; Gong et al., 2006; Li et al., 2007; Szalma et al., 2007; Ribaut et al., 1996, 2007; Hu et al., 2008; Wu et al., 2008), our study differed in many aspects, and these differences included parents, populations and ecological environments (Table 5). Previous studies focused on the $\mathrm{F}_{2}$ populations, where this kind of population has a deficiency named temporality, since there are no continued plants for DNA extraction and phenotypic observation. Conversely, the RIL population in our study is immortal and can be used again and again in different regions or time. More meaningfully, the QTLs identified in our study were different from the previous ones in number, chromosomal position and genetic effects, and these differences could probably be explained by differences in parental lines, segregation population or ecological conditions. Moreover, previous ecological conditions involved different locations and water content in soil (Ribaut et al., 1996; Khairallah et al., 1998; Zhang et al., 2004; Tang et al., 2005; Gong et al., 2006; Hu et al., 2008; Wu et al., 2008), whereas different $\mathrm{N}$ regimes were not used to map QTLs affecting DTS, except in the study by Ribaut et al. (2007).

\begin{tabular}{|c|c|c|c|}
\hline Reference & Parents & Population & Environments \\
\hline Ribaut et al., 1996 & $\mathrm{Ac} 7643 \mathrm{~S}_{5}, \mathrm{Ac} 729 / \mathrm{TZSRWS}_{5}$ & $\mathrm{~F}_{2}$ & Three water regimes \\
\hline Khairallah et al., 1998 & Ki3, CML139 & $\mathrm{F}_{2}^{2}$ & Two locations \\
\hline Zhang et al., 2004 & L050, B73 & $\mathrm{F}_{2}^{2}$ & Well water, drought stress \\
\hline Tang et al., 2005 & Zong3, 87-1 & $\mathrm{F}_{2}^{2}$ & Two locations \\
\hline Gong et al., 2006 & GN165, Dan330 & $\mathrm{F}_{2}^{2}$ & Two locations \\
\hline Li et al., 2007 & Dan340, N04 & $\mathrm{F}_{2}^{2}$, backcross & One location \\
\hline Ribaut et al., 2007 & Ac7643S5, Ac7729/TZSRWS5 & $\mathrm{F}_{2}, \mathrm{C}$ & Two $\mathrm{N}$ regimes \\
\hline Szalma et al., 2007 & Tx303, B73 & Near-isogenic lines & Two years \\
\hline Hu et al., 2008 & Qiong68, K12 & $\mathrm{F}_{2}$ & Two locations \\
\hline Wu et al., 2008 & A188, 91Huang 15 & $\mathrm{~F}_{2}^{2}$ & Two water regimes \\
\hline This study & Mo17, Huangzao4 & RIL & Two $\mathrm{N}$ regimes \\
\hline
\end{tabular}

$\mathrm{RIL}=$ recombinant inbred line.

Although different $\mathrm{N}$ regimes were also used to map the QTLs for DTS by Ribaut et al. (2007), our experiment differed from theirs in many aspects (Table 6). They found QTLs on chromosomes 1,2, 4, and 8, while we could not detect any QTLs on these chromosomes. These authors did map one QTL to chromosome 9, but a total of three QTLs were located in our study. These differences are probably due to different parents, populations or types of genetic map markers. 
Table 6. Quantitative trait loci (QTLs) identified under different nitrogen regimes.

\begin{tabular}{lllll}
\hline Reference & Parents & Population & Marker type & QTL number (Chr. No.) \\
\hline Ribaut et al., 2007 & Ac7643S5, Ac7729/TZSRWS5 & $\mathrm{F}_{2}$ & RFLP & Seven $(12,4,4,6,8,9)$ \\
This study & Mo17, Huangza04 & RIL & SSR & Four $(6,9,9,9)$ \\
\hline RIL = recombinant inbred line. & &
\end{tabular}

$\mathrm{RIL}=$ recombinant inbred line.

Moreover, the QTL named $Q 9 b-h n$ in our study was quite near Nc134, with a mapping interval of $0 \mathrm{cM}$, which suggested that the linked marker Nc134 could probably be co-segregated with the gene controlling DTS within the QTL and, thus, could be considered as a candidate marker in MAS in a maize breeding program. The other three QTLs in our results had an over 1-cM mapping interval corresponding to the nearest markers. For this, we could add some other molecular markers to these given chromosomal regions to map the QTLs more precisely, and a further research is in progress based on the constructed RIL population and genetic map.

\section{ACKNOWLEDGMENTS}

Research supported by the Science and Technology Bureau of Sichuan Province (\#2007q14-029), P.R. China.

\section{REFERENCES}

Agrama HAS, Zakaria AG, Said FB and Tuinstra M (1999). Identification of quantitative trait loci for nitrogen use efficiency in maize. Mol. Breed. 5: 187-195.

Bänziger M, Betran FJ and Lafitte HR (1997). Efficiency of high-nitrogen selection environments for improving maize for low-nitrogen target environments. Crop Sci. 37: 1103-1109.

Doerge RW and Churchill GA (1996). Permutation tests for multiple loci affecting a quantitative character. Genetics 142 : 285-294.

Gong Q, Wang TY, Tan XL, Shi YS, et al. (2006). QTL analysis of traits related to flowering in elite maize inbred line Dan330 with early maturity. J. Plant Genet. Resour. 7: 437-441.

$\mathrm{Hu}$ YM, Wu X, Li CX, Fu ZY, et al. (2008). Genetic analysis on the related traits of florescence for hybrid seed production in maize. J. Nanjing Agric. Univ. 31: 11-16.

Khairallah MM, Bohn M, Jiang C, Deutsch JA, et al. (1998). Molecular mapping of QTL for southwestern corn borer resistance, plant height and flowering in tropical maize. Plant Breed. 117: 309-318.

Li YL, Li XH, Dong YB, Niu SZ, et al. (2007). QTL mapping of developmental stages using $\mathrm{F}_{2: 3}$ and $\mathrm{BC}_{2} \mathrm{~S}_{1}$ populations derived from the same cross in maize. Acta Agric. Boreali-Sin. 22: 38-43.

Liu XH, Tan ZB and Tan ZB (2009). Molecular mapping of a major QTL conferring resistance to SCMV based on immortal RIL population in maize. Euphytica 167: 229-235.

Liu X, Zheng Z, Tan Z, Li Z, et al. (2010). QTL mapping for controlling anthesis-silking interval based on RIL population in maize. Afr. J. Biotechnol. 9: 950-955.

McIntyre CL, Mathews KL, Rattey A, Chapman SC, et al. (2010). Molecular detection of genomic regions associated with grain yield and yield-related components in an elite bread wheat cross evaluated under irrigated and rainfed conditions. Theor. Appl. Genet. 120: 527-541.

Ribaut JM, Hoisington DA, Deutsch JA, Jiang C, et al. (1996). Identification of quantitative trait loci under drought conditions in tropical maize. 1. Flowering parameters and the anthesis-silking interval. Theor. Appl. Genet. 92: 905-914.

Ribaut JM, Fracheboud Y, Monneveux P, Banziger M, et al. (2007). Quantitative trait loci for yield and correlated traits under high and low soil nitrogen conditions in tropical maize. Mol. Breed. 20: 15-29.

Sabadin PK, Souza CL Jr, Souza AP and Garcia AAF (2008). QTL mapping for yield components in a tropical maize population using microsatellite markers. Hereditas 145: 194-203.

Szalma SJ, Hostert BM, Ledeaux JR, Stuber CW, et al. (2007). QTL mapping with near-isogenic lines in maize. Theor. Appl. Genet. 114: 1211-1228.

Tang H, Yan JB, Huang YQ, Zheng YL, et al. (2005). QTL mapping of five agronomic traits in maize. Yi. Chuan Xue. 
Bao. 32: 203-209.

Voorrips RE (2002). MapChart: software for the graphical presentation of linkage maps and QTLs. J. Hered. 93: 77-78.

Wan XY, Wan JM, Jiang L, Wang JK, et al. (2006). QTL analysis for rice grain length and fine mapping of an identified QTL with stable and major effects. Theor. Appl. Genet. 112: 1258-1270.

Wang S, Basten CJ and Zeng ZB (2010). Windows QTL Cartographer 2.5. Department of Statistics, North Carolina State University, Raleigh. Available at [http://statgen.ncsu.edu/qtlcart/WQTLCart.htm]. Accessed March 10, 2010.

Wu JW, Liu C, Wang TY, Li Y, et al. (2008). QTL analysis of flowering related traits in maize under different water regimes. J. Maize Sci. 16: 61-65.

Yang GB, Liu XY, Gao DJ, Tan FZ, et al. (2007). Constrict factors and countermeasures of maize planting in northern premature areas of Heilongiiang. Heilongjiang Agric. Sci. 6: 18-19.

Yang X, Guo Y, Yan J, Zhang J, et al. (2010). Major and minor QTL and epistasis contribute to fatty acid compositions and oil concentration in high-oil maize. Theor. Appl. Genet. 120: 665-678.

Zhang JM, Liu C, Shi YS, Song YC, et al. (2004). QTL analysis of parameters related to flowering in maize under drought stress and normal irrigation condition. J. Plant Genet. Resour. 5: 161-165. 